Document downloaded from:

http://hdl.handle.net/10251/146494

This paper must be cited as:

Benlloch Baviera, JM.; González Martínez, AJ.; Pani, R.; Preziosi, E.; Jackson, C.; Murphy, J.; Barbera Ballester, J.... (2018). The MINDVIEW project: First results. European Psychiatry. 50:21-27. https://doi.org/10.1016/j.eurpsy.2018.01.002

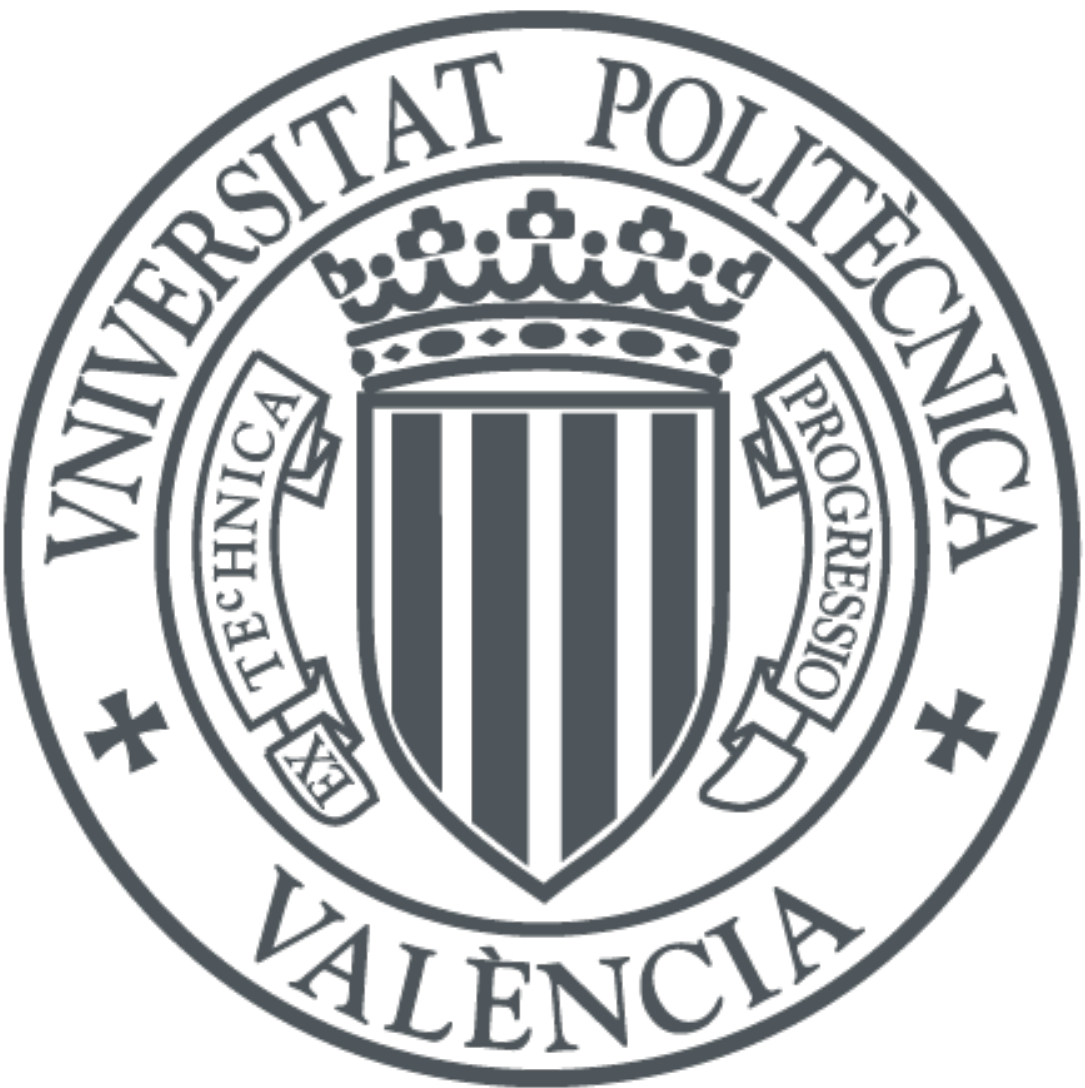

The final publication is available at

https://doi.org/10.1016/j.eurpsy.2018.01.002

Copyright Elsevier

Additional Information 


\title{
The MINDVIEW Project: First Results
}

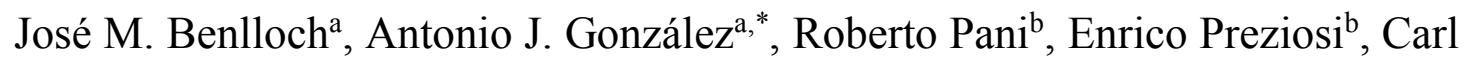 \\ Jackson $^{\mathrm{c}}$, John Murphyc ${ }^{\mathrm{c}}$, Julio Barberád, Carlos Correcher ${ }^{\mathrm{d}}$, Sebastian Aussenhofere, \\ Daniel Gareis ${ }^{\mathrm{e}}$, Dimitris Visvikis ${ }^{\mathrm{f}}$, Julien Bert ${ }^{\mathrm{f}}$, Bengt Langstrom ${ }^{\mathrm{g}}$, Lars Farde ${ }^{\mathrm{h}, \mathrm{i}}$, \\ Miklos Toth $^{\mathrm{h}}$, Jenny Haggkvist ${ }^{\mathrm{h}}$, Fabio V. Caixeta ${ }^{\mathrm{j}}$, Klas Kullander ${ }^{\mathrm{j}}$, Ian Somlai- \\ Schweigerk $^{\mathrm{k}}$, Markus Schwaigerk \\ ${ }^{a}$ Institute for Instrumentation in Molecular Imaging (I3M), Universidad Politécnica de Valencia - CSIC, Valencia, SPAIN \\ ${ }^{b}$ Department of Molecular Medicine, Sapienza University of Rome, ITALY \\ ${ }^{c}$ SensL Technologies, Cork, IRELAND \\ ${ }^{d}$ Oncovision, Valencia, SPAIN \\ ${ }^{e}$ NORAS MRI products GmbH, Hochberg, GERMANY \\ ${ }^{f}$ INSERM, UMR1101, LaTIM, Université de Bretagne Occidentale, Brest, FRANCE \\ ${ }^{g}$ BENCAR, Uppsala, SWEEDEN \\ ${ }^{h}$ Dept. of Clinical Neuroscience, Centre for Psychiatry Research, Karolinska Institutet and Stockholm County Council, Stockholm, SWEDEN \\ ${ }^{i}$ Precision Medicine \& Genomics, AstraZeneca, PET Science Center, Karolinska Institutet, Stockholm, SWEEDEN \\ ${ }^{j}$ Department of Neuroscience, Uppsala University, Uppsala, SWEEDEN \\ ${ }^{k}$ Technische Universität München, Dept. of Nuclear Medicine, Munich, GERMANY \\ *Corresponding author: agonzalez@i3m.upv.es
}

Elsevier use only: Received date here; revised date here; accepted date here

\begin{abstract}
We present the first results of the MINDVIEW project. An innovative imaging system for the human brain examination, allowing simultaneous acquisition of PET/MRI images, has been designed and constructed. It consists of a high sensitivity and high resolution PET scanner integrated in a novel, head-dedicated, radio frequency coil for a 3T MRI scanner. Preliminary measurements from the PET scanner show sensitivity 3 times higher than state-of-the-art PET systems that will allow safe repeated studies on the same patient. The achieved spatial resolution, close to $1 \mathrm{~mm}$, will enable differentiation of relevant brain structures for schizophrenia. A costeffective and simple method of radiopharmaceutical production from ${ }^{11} \mathrm{C}$-carbon monoxide and a mini-clean room has been demonstrated. It has been shown that ${ }^{11} \mathrm{C}$-raclopride has higher binding potential in a new VAAT null mutant mouse model of schizophrenia compared to wild type control animals. A significant reduction in TSPO binding has been found in gray matter in a small sample of drug-naïve, first episode psychosis patients, suggesting a reduced number or an altered function of immune cells in brain at early stage schizophrenia.

PACS:

Keywords: Schizophrenia, Positron Emission Tomography, Silicon photomultipliers and Magnetic Resonance.
\end{abstract}




\section{Introduction}

Currently, the two main functional imaging modalities, functional MRI (fMRI) and Molecular Imaging (MI), cannot be used to clinically diagnose schizophrenia nor depression, two major mental disorders, for a variety of reasons. MRI and fMRI have been shown to differentiate diagnosed schizophrenia from healthy controls only on a statistical basis within a population sample but not on individual basis. Molecular Imaging (especially PET, Positron Emission Tomography) is an extremely useful technique for understanding the pharmacological treatment of schizophrenia and severe depression and it has helped developing the most recent generation of effective drugs. However, the utility of PET imaging for the clinical diagnosis of mental disorders is practically limited by: the significant high cost, the complexity of the infrastructure required to generate radiopharmaceuticals and the limited sensitivity and resolution of current scanners. Unfortunately, the recent advent of PET/MR has not improved the situation on psychiatric disorders since some limitations of PET technology remain unsolved:

- PET scanner sensitivity is still poor, not allowing follow-up studies on the same patient to be safely performed due to the high radioactive dose necessary.

- PET scanner resolution does not allow the visualization of brain critical structures. The standard PET/CT and the new PET/MRI whole body scanners result in a PET image resolution in the 4-5 mm FWHM range and, therefore, they are not optimized for imaging critical structures of the brain with dimensions of few millimetres. Even for processes localized within the amygdala, a part of the limbic system related to fear and anxiety, it is currently not possible to differentiate if they are occurring within the basolateral complex or the central nucleus $[1]$.

- Current PET/MRI scanners are extremely expensive, hampering a wide expansion of this technology.

- The infrastructure required to synthesize radiotracers is still complex, expensive and occupies a large space. Moreover, most new radiopharmaceuticals require a new expensive specific module, in a hot cell. Therefore, 
although some radiopharmaceuticals have the potential for clinical use in relation to mental disorders, none is actually used in the clinical practice.

The dopamine system has since long been of central interest in schizophrenia research. Over the years, PET imaging has shown evidence for abnormalities in the brain dopaminergic system [2] and that clinically useful treatments interact with dopamine receptors in the central nervous system [3]. The imaging findings support the view that striatal dysregulation of dopamine neurotransmission may be central to the disruption of neuronal networks and symptoms of schizophrenia.

Apart from the striatum, the dopamine subsystems also innervate several small brain structures in which there is a limited expression of dopaminergic markers, for instance the amygdala, the entorhinal cortex and subnuclei of the thalamus. Considering the hierarchical organization of the brain it cannot be excluded that some of these structures may have a key role in the pathophysiology of schizophrenia. The advent of PET systems having a resolution of about $1 \mathrm{~mm}$ will thus provide a significant improvement for such examinations.

Beyond dopamine, present evidence suggests alterations in other major neurotransmission systems in neuropsychiatric disorders including schizophrenia. For instance, the glutamate hypothesis arose initially from observations that administration of ketamine and other non-competitive antagonists at the glutamatergic NMDA receptor (NMDAR) leads to psychological effects, which closely resemble symptoms that occur in schizophrenia [4]. Interplay between hippocampal glutamate and striatal dopamine systems has recently been found to be disturbed in subjects at risk of psychosis, with the degree to which it is changed being related to the risk of transition to psychosis [5]. Among the metabotropic glutamate receptors, mGluR5 is of particular interest as it shares a functional link with the NMDAR [6]. Importantly, several radioligands have been developed for PET imaging of the mGluR5 receptor in humans [7][8][9]. However, none has to our knowledge been applied to schizophrenia research so far.

Beyond the neurotransmission systems, several lines of evidence are indicative of a role for immune activation in the pathophysiology of schizophrenia. However, studies using PET and radioligands for the translocator protein (TSPO), a marker for glial activation, have yielded inconsistent results. Importantly, patients 
investigated in initial studies have been on antipsychotic medication, i.e. compounds that may dampen immune cell activity.

\section{The MINDVIEW Project}

MINDVIEW (Multimodal Imaging of Neurological Disorders) is a European project addressing the topic: "Development of effective imaging tools for diagnosis, monitoring and management of mental disorders". It is therefore a project of a multidisciplinary nature. The main objective of MINDVIEW is the design and construction of an innovative high sensitivity and resolution PET/MRI scanner, dedicated to the brain examination, for the study of psychiatric disorders in a clinical research setting, and its validation and preliminary application in clinical populations for the diagnosis and monitoring of schizophrenia.

Another goal is the development of a mini radiopharmaceutical platform that will trigger an expansion of PET technology by facilitating the use of new compounds and by reducing laboratory costs. Furthermore, this tool will be essential for the assurance of same quality production and protocols in multi centric clinical trials and make new radiopharmaceuticals more broadly available for specific imaging of neurotransmitters (endogenous and exogenous) pathways relevant for schizophrenia and depression disorders. Finally, it is also an objective of MINDVIEW the development of reliable animal models to be used for the validation of new radiopharmaceuticals for the diagnosis of schizophrenia.

\section{Materials}

In this section, we describe several tools that have been developed within the MINDVIEW project: an innovative brain dedicated PET/MRI scanner and a new mini clean room for radiopharmaceutical production.

\subsection{MINDVIEW Integrated PET-RF Coil Imager}

The MINDVIEW approach to achieve simultaneous PET/MRI imaging is based on the development of a brain PET imager integrated into a purposely designed head dedicated transmitter/receiver (TR) RF coil system. Therefore, MINDVIEW takes advantage of current MRI scanners and upgrades them with a brain dedicated 
PET/RF device. The dimensions of the full PET/RF system represent a balance between PET and RF performance as well as patient comfort, resulting into a portable and compact design.

The new head dedicated RF coil system has a birdcage TR design, to allow patient vision between the 16 coil rungs, providing a geometric aperture of $26 \mathrm{~cm}$, ample for comfortable head allocation [10]. In order to minimize distortions in the MRI performance, the distance between the PET shielding and the RF rungs conductor is about $3 \mathrm{~cm}[11]$.

The PET subsystem consists of 3 rings of 20 detector modules per ring (Fig. 1), leaving a geometrical aperture of $\sim 33 \mathrm{~cm}$ in diameter to allow enough vision to schizophrenia patients. Each detector module is made of a single LYSO crystal $(5 \mathrm{~cm} \times 5 \mathrm{~cm} \times 2 \mathrm{~cm})$, a SiPM (Silicon Photomultiplier) photo-sensor array and the related frontend electronics. The 3 rings cover an axial field of view (FOV) of 15,2 cm. Coincidences of gamma rays between one PET detector module and any of the 9 opposite modules produces a transaxial FOV of about $24 \mathrm{~cm}$, which ensures full coverage of the human brain [12].

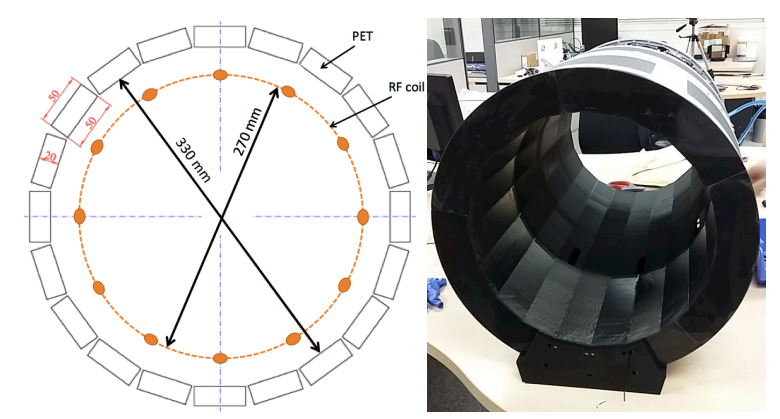

Figure 1. Left, sketch of the PET ring. Right, front photograph of the PET insert in the lab bench.

Achieving $1 \mathrm{~mm}$ spatial resolution in the central brain region with PET detectors close to the object, such as in MINDVIEW, is not trivial and requires depth of interaction (DOI) determination of the gamma rays inside the thick scintillating crystals, to avoid blurring produced by parallax error. Two major LYSO scintillator crystal configurations have been considered: a staggered pixelated crystal array and a monolithic approach [13]. There are advantages and disadvantages for each configuration that have been described in the literature [14][15]. We decided to build the prototype using monolithic blocks, as detailed elsewhere [13]. For monolithic crystal 
designs, several DOI schemes have been proposed based on the correlation between the DOI and the measured light distribution [16][17][18][19][20].

Different crystal surface treatments have been proposed to optimize the performance of detectors based on thick monolithic crystals: specular reflectors, black absorbent paint or white Teflon [21]. The final block treatment design for our PET system uses black painted lateral walls combined with a retroreflector (RR) layer [19] on the $50 \mathrm{~mm} \times 50 \mathrm{~mm}$ entrance surface (Fig. 2).

The light coming from each scintillating crystal is detected using high-density, custom designed, SiPM arrays from SensL (MINDVIEW-Series type, similar to the now-in-production J-series technology) [22]. New developments in SiPM fabrication technology and packaging were utilized. SensL engineered a low defect active area into the CMOS foundry process. This resulted in a dark count reduction from $744 \mathrm{kHz} / \mathrm{mm}^{2}$ in previous SiPM devices to a value of $30 \mathrm{kHz} / \mathrm{mm}^{2}$ at typical room temperatures. To provide MRI compatibility, a custom through-silicon-via (TSV) process \& package was developed. This wafer scale package uses no ferromagnetic material in its construction. The photosensor matrix is composed of 144 SiPMs ( $35 \mu \mathrm{m}$ cell size), arranged in 12 by 12 columns and rows, covering an active area of approximately $51 \mathrm{~mm} \times 51 \mathrm{~mm}$ (Fig. 2). The SiPM package was tested inside high magnetic fields with no measured change in performance [23]. Each individual SiPM presents an active area of $3 \mathrm{~mm} \times 3 \mathrm{~mm}$ with $4.36 \mathrm{~mm}$ pitch, and is typically operated at a bias voltage of about $31 \mathrm{~V}, 6,5 \mathrm{~V}$ over breakdown voltage. 


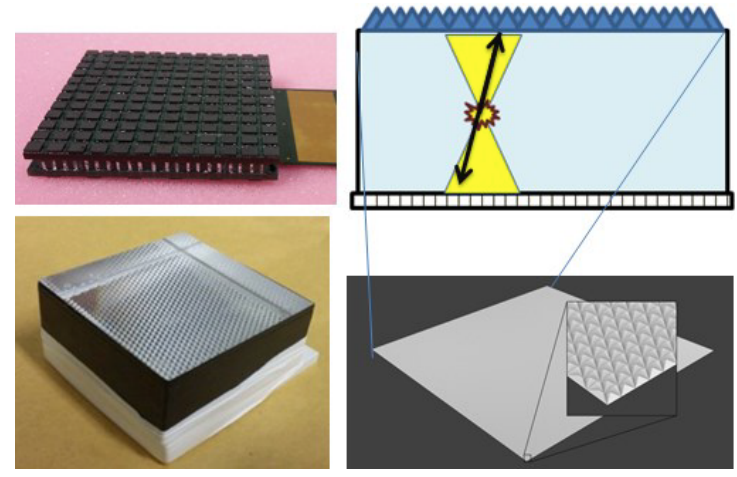

Figure 2. Top-left, photograph of the custom 12x12 SiPM array. Bottom-left, photograph of the $20 \mathrm{~mm}$ thick crystal showing a RR across the scintillator. Right, sketch of the monolithic block and the expected light distribution.

Each SiPM array is directly connected to a readout circuit that provides outputs for each row and column of the array [19]. This readout scheme significantly reduces the number of channels to be digitized, while allowing accurate sampling of the scintillation light distribution produced by gamma photon interactions in the monolithic crystal. The 24 row and column signals are digitized by custom ADC boards (12-bit precision).

A special algorithm that uses the information on the integral and the maximum of the scintillation light distribution has been developed. It allows fast and efficient DOI determination of the gamma ray in a continuous way [20][24]. In order to improve the determination of the 3D position of interaction of gamma-photons in the crystal, a simplified algorithm customized for rows and columns readout, and able to take into account the DOI information, has been implemented. This DOI-dependent [20] algorithm, named RTP [25], corrects the error in position estimation for gamma rays that interact close to the crystal edges, for which a cut-off of the scintillation light distribution occurs.

\subsection{Mini Clean Room and Labelling Synthesis Platforms for Radiopharmaceutical Production}

A mini clean room that simplifies GMP production and makes it more reliable and cheaper, aiming for an easy access to various tracers, has been developed. The mini clean room concept can be adjusted to the platform technology used and allows protocols to keep a GMP environment in control. In this project, the focus has then been to develop tracer technology on new platforms, mainly on the preparation of ${ }^{11} \mathrm{C}$-labelled tracers, which is 
adapted to the mini clean room concept in order to make the tracer production more applicable [26]. In particular, the use of ${ }^{11} \mathrm{C}$-carbon monoxide in new applications [26] and on new technology platforms [28] has been developed. Both ${ }^{11} \mathrm{C}$-methyl iodide or ${ }^{11} \mathrm{C}$-carbon monoxide were used in the labelling of an established dopaminergic tracer like Raclopride, but now labelled in various positions to show the flexibility and potential of the labelling strategies explored. Furthermore, we have re-examined production methods for ${ }^{11} \mathrm{C}$-methyl iodide [29] applied to these new technology platforms. In a monkey study, ${ }^{11} \mathrm{C}$-methyl iodide and ${ }^{11} \mathrm{C}-\mathrm{CO}$ were used to produce ${ }^{11} \mathrm{C}$-raclopride labelled in 2 different positions, exploring its impact on the images due to differences in metabolism [30]. The use of ${ }^{11} \mathrm{C}$-carbon monoxide is opening up new avenues to label many potential tracers.

\section{Results}

\subsection{Performance Measurements of the MINDVIEW Scanner}

Performance tests of the PET detectors were carried out both with non-encapsulated radioactive sources and with FDG filled phantoms. We found detector resolution (FWHM) values ranging from $1.7 \mathrm{~mm}$ on average at the entrance layer (20-15 $\mathrm{mm}$ from photosensor), to $0.7 \mathrm{~mm}$ at the layer nearest to the photosensor [19]. The RR layer tends to return an energy resolution that slightly depends on the interaction depth. The overall energy resolution is better than $13 \%$. We determined an average DOI resolution around $4 \mathrm{~mm}$ with a continuous response and excellent linearity, making it possible to efficiently correct for the parallax error using such thick monolithic crystals.

A solution of FDG with an activity of about $150 \mu \mathrm{Ci}$ was filled in a Derenzo-like phantom, and acquired during 20 min after positioning near the CFOV [31]. The phantom has several groups of rods with diameters ranging from $1.2 \mathrm{~mm}$ to $4.8 \mathrm{~mm}$.

Fig. 3 shows on the left the FBP-STIR-3D algorithm with $0.8 \mathrm{~mm}$ virtual pixel size and $0.4 \mathrm{~mm}$ voxels. On the right, we show the reconstructed image using LM-OS with virtual pixels of $1 \mathrm{~mm}$ and $0.5 \mathrm{~mm}$ voxels. Here, 100 subsets and 1 iteration were used. Scatter and random corrections were applied but not attenuation 
correction. In both methods, it can be observed that the $1.6 \mathrm{~mm}$ rods can be visualized albeit with a limited signal to noise ratio.

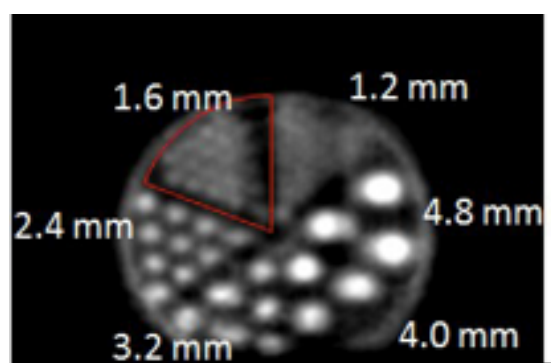

Figure 3. Reconstructed images of a Derenzo-like phantom filled with about $150 \mu \mathrm{Ci}$ of FDG and scanned during 20 min: (left) shows the reconstruction using FBP STIR-3D; (right) shows the LM-OS reconstruction.

An alternative approach based on the use of an Iterative Random IDRF Sampling (IRIS) projector [32] can further improve results. This previously proposed projector has been modified for monolithic crystal based detectors modeling the response associated with a given line of response. Implemented within an iterative reconstruction, it has the potential to further improve image resolution and signal to noise ratio performance as shown in the simulated Derenzo phantom images in Fig. 4. Scanner sensitivity, measured with a point source at the center of the FOV, is $2,7 \%$.

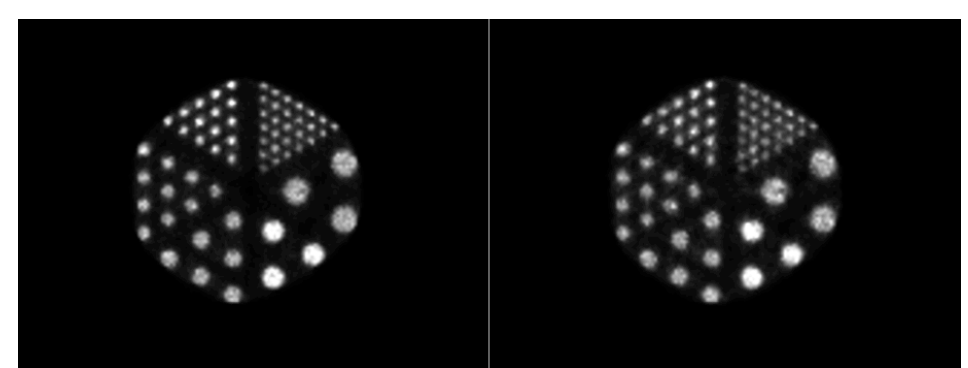

Figure 4. LM-OSEM reconstructed images of simulated MINDVIEW datasets of a Derenzo phantom using (left) the first interaction coordinates in the crystal (ideal case ground truth), and (right) the IRIS projector (IDRF estimated using twenty samples for each line of response).

The PET scanner has been tested within the MR system of the hybrid PET-MR from Siemens (mMR) at Klinikum Rechts der Isar in Munich, finding no significant interference from the MRI working at very demanding gradient duty cycle sequences. In a different test, several MRI images (see Fig. 5) were acquired from the head of a patient with the hybrid device, with a dummy version of the PET system. 


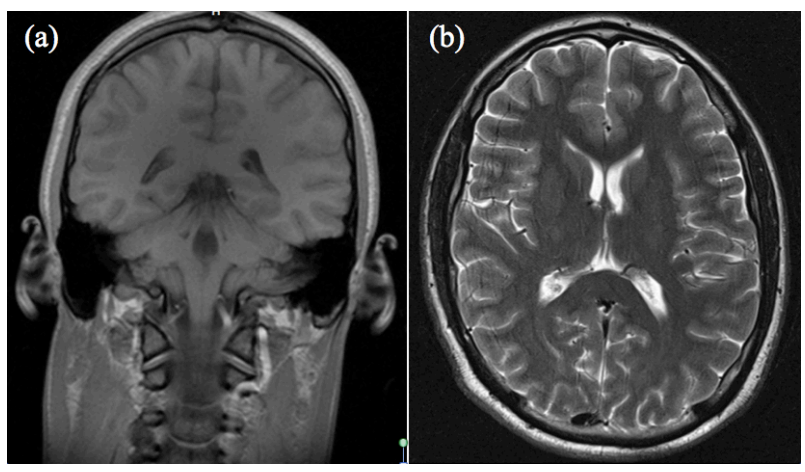

Figure 5. Reconstructed MRI images with the MINDVIEW RF coil hybrid prototype. (a) T1 weighted fast low angle shot, TR 250 , TE 2.49 , slice thickness $4 \mathrm{~mm}$, number of averages 1 , matrix $256 \times 320$, flip angle $70^{\circ}$, field of view $220 \times 220$. and (b) T2 weighted turbo spin echo, TR 6000 , TE 100 , slice thickness $4 \mathrm{~mm}$, number of averages 1 , matrix $512 \times 512$, flip angle $150^{\circ}$, field of view $220 \times 220$.

\subsection{Establishing an Animal Model with Altered Sensorimotor Gating}

To validate and extend on the use of radiotracers, we established a mouse model that affects the aminergic systems of the brain. Specifically, we used mice that had the allele encoding the vesicular aminergic associated transporter VAAT, or Slc10A4, deleted [33][34]. VAAT adds functionality to aminergic neurons through a mechanism involving presynaptic vesicles. VAAT null mutant mice have been shown to develop normally without obvious abnormalities and normal motor function in spite of defective cholinergic synaptic transmission at the neuromuscular junction [35]. However, VAAT null mutant mice show clear alterations in aminergic homeostasis, have increased susceptibility to a cholinergic form of status epilepticus [33] and are hypersensitive to dopaminergic stimulants such as amphetamine [34]. Moreover, decreased dopamine uptake has been observed in vesicle preparations from VAAT null mutant mice, while over expression of VAAT resulted in increased acidification of synaptic vesicles. These alterations led us to hypothesize that VAAT null mutants were affected in systems where aminergic functionality is a prerequisite, such as in behaviours related to the symptoms of schizophrenia. For this purpose, we here investigated whether the deletion of the VAAT gene in mice would 
alter sensorimotor gating, similar to the deficient sensorimotor gating seen in the dopamine overactivity rodent model of schizophrenia [36].

Mice were analysed in different pre-pulse inhibition regimens; high amplitude (20 db above back ground noise) and short vs long inter-stimuli intervals (Fig. 6). Mice that were either wild-type (WT, n=17), VAAT knock-outs $(\mathrm{KO}, \mathrm{n}=21)$ or overexpressing the VAAT gene (NSE, $\mathrm{n}=13)$ displayed either normal or enhanced sensorimotor gating responses in the pre-pulse inhibition (PPI) test at $30 \mathrm{~ms}$ inter-stimuli intervals (short IEI) at $85 \mathrm{~dB}$ (high amp) pre-stimulus amplitudes. At $100 \mathrm{~ms}$ inter-stimuli intervals (long IEI) VAAT null mutant mice show increased sensorimotor gating. Mice overexpressing VAAT (Slc10a4 NSE mice) showed responses similar to wild type (WT) littermate controls. VAAT null mutant animals had a higher ability to filter, process and link the pre-pulse and startle pulse compared to control animals. In contrary, mice overexpressing Slc10a4 did not show any differences in the PPI test. Such mice were constructed to overexpress Slc10a4 under the neuronal specific enolase promoter, Slc10a4-NSE [34]. Our results suggest that VAAT null mutant mice may be used as a small animal schizophrenia model based on their response to the pre-pulse inhibition (PPI) test.

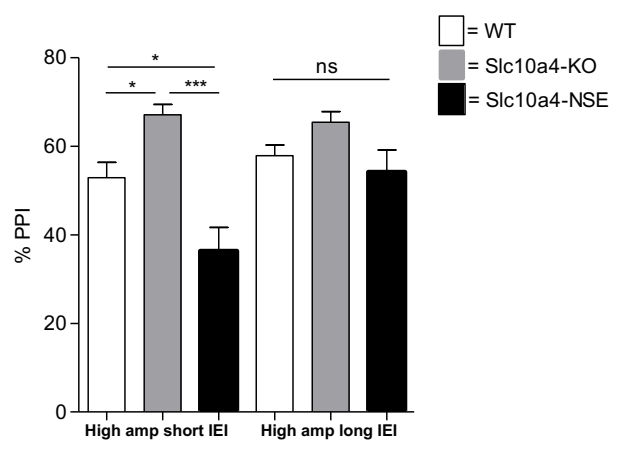

Figure 6. Evaluation of mice lacking VAAT or overexpressing VAAT (Slc10A4) in the PPI test. PPI values are shown as percentages of the average startle response measured at the beginning of each session of each animal $\pm \mathrm{SEM}, \mathrm{nWT}=17, \mathrm{nKO}=21, \mathrm{nNSE}=13$ animals. Statistic analyses by one-way ANOVA followed by Tukey's MCT (GraphPad Prism 5) *p $<0.05,{ }^{*} \mathrm{p}<0.01,{ }^{* * *} \mathrm{p}<0.001$. 


\subsection{Validation of Radiopharmaceuticals with the Schizophrenia Animal Model}

We next evaluated uptake and distribution of radiopharmaceuticals in VAAT null mutant mice using established and novel radioligands in PET. We used the dopamine $\mathrm{D}_{2}$ antagonist ${ }^{11} \mathrm{C}$-raclopride, which is a reliable and robust radioligand with well characterized properties in small animal models. Importantly, ${ }^{11} \mathrm{C}$ raclopride has since long been used in clinical studies to evaluate patients with schizophrenia [37][1]. In addition to dopamine, serotonin has also been associated to schizophrenia [38]. Thus, we also used the novel serotonin 2A (5-HT2A) receptor agonist ${ }^{11} \mathrm{C}$-Cimbi [39]. On the same experimental day, two PET measurements with ${ }^{11} \mathrm{C}$ raclopride and ${ }^{11} \mathrm{C}$-Cimbi, respectively, were conducted in each animal. We imaged four wild type mice and five VAAT null mutant mice with ${ }^{11} \mathrm{C}$-raclopride. Three wild type mice and five VAAT null mutant mice were imaged with ${ }^{11} \mathrm{C}$-Cimbi. In a preliminary analysis, we found that Slc10a4 null mutant mice have higher binding potential of ${ }^{11} \mathrm{C}$-raclopride compared to wild type control animals (Fig. 7) whereas no such evident effect could be observed for ${ }^{11} \mathrm{C}$-Cimbi binding.

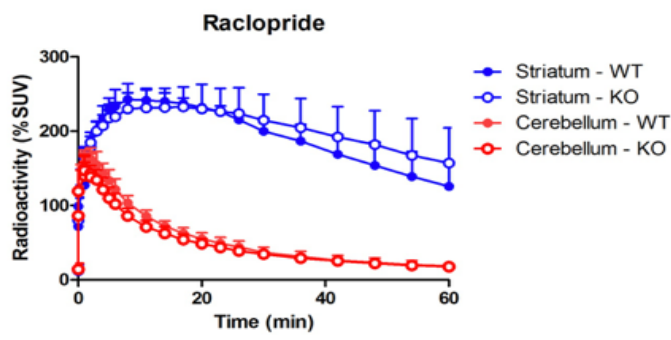

$\left[{ }^{11} \mathrm{C}\right]$ Raclopride

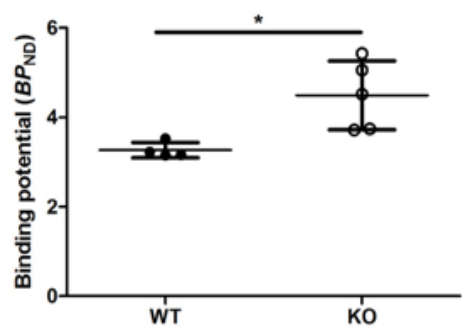

Figure 7. Radiotracer time activity and binding potential. Top, average Time Activity Curves in the striatum and cerebellum for ${ }^{11} \mathrm{C}$-Raclopride. Bottom, binding potential $B P_{\mathrm{ND}}$ of wild type control mice (WT, $\mathrm{n}=4)$ and VAAT null mutant mice $(\mathrm{KO}, \mathrm{n}=5)$ measured in the striatum. 


\subsection{Clinical findings in schizophrenia studies}

At the Karolinska Institutet there have recently been examined 16 drug-naïve, first episode psychosis patients and 16 healthy controls using PET and the TSPO radioligand ${ }^{11} \mathrm{C}-\mathrm{PBR} 28$ [40]. There was a significant reduction of ${ }^{11} \mathrm{C}-\mathrm{PBR} 28 \mathrm{VT}$ in patients compared to healthy controls in gray matter (GM) as well as in secondary regions of interest. In this limited sample, there was no correlation between GM binding and clinical or cognitive measures after correction for multiple comparisons. The observed decrease in TSPO binding suggests reduced number or altered function of immune cells in brain in early stage schizophrenia.

\section{Discussion}

Increasing the sensitivity of PET scanners is mandatory for routine imaging diagnosis and follow-up of patients with mental disorders. One way of increasing the sensitivity of current PET scanners is by augmenting significantly the axial FOV, which implies a very high associated cost. The approach followed by the MINDVIEW project, i.e. integrating the PET system with the RF coil, has the advantage of increasing significantly the sensitivity of the scanner for the brain examination with a reduced number of detector modules. This alternative method of increasing the PET sensitivity has the potential of further extending the PET/MRI technique through upgrading the large MRI installed base with a cost-effective and portable brain PET/RF scanner.

PET images were obtained with phantoms while the MRI system was working at highly demanding pulse sequences, which demonstrates that no major interference was found in the PET system. On the other hand, high quality MRI images were obtained with the RF coil of the integrated hybrid system. Simultaneous PET/MRI images with patients are expected early 2018.

From the study on the binding potential with WT and VAAT mice it could be concluded that the alterations found in the monoamine homeostasis are more readily detectable with Raclopride than with Cimbi. Whether this is due to a larger effect on the dopaminergic system in Slc10a4 mutant mice or to the properties of the radiotracers used in this study remains to be established. For the latter, we are devising an approach where we 
specifically target the serotonergic system, in which we should be able to discriminate serotonergic specific effects.

The clinical finding that reduced numbers or altered function of immune cells in brain appears in early stage schizophrenia is very relevant. However, the study also illustrates the problems in recruiting a large sample of first-episode medication free patients with schizophrenia. Despite a clinical research organization covering a large catchment area of Northern Stockholm it took 4 years to recruit the 16 patients. In order to recruit larger patient samples future research would therefore benefit significantly from multi-center studies using more widely available PET-systems, that may even be shipped on a nation-wide basis.

In addition, schizophrenia research would benefit from the potential to examine each patient with two or more radioligands targeting different neurotransmission systems or functional markers such as TSPO. Ideally these studies should be repeated during different states of the disorder. The sensitivity of the next generation PET-systems will be critical to allow for such extended protocols within as low as reasonably acceptable limits of radiation exposure.

\section{Conclusions}

Several important tools have been developed during the MINDVIEW project for the diagnosis and follow up of schizophrenia disease. In particular, an innovative and cost-effective 1,6 $\mathrm{mm}$ spatial resolution, over the entire FOV, PET scanner has been developed, which has been integrated with a head dedicated RF coil. As stated in the introduction, it is estimated that a PET spatial resolution of $\sim 1 \mathrm{~mm}$ is required to answer specific questions relating to brain function in small brain regions and improve the diagnostic potential of the images. Therefore, we have achieved the required resolution, and at the same time increased the sensitivity, by designing a method to provide good DOI information, which is critical due to the close proximity to the brain.

The high sensitivity of the PET scanner of 2,7\% (state-of-the-art whole body PET systems provide sensitivities close to $1 \%$ ) should allow for longitudinal examinations on the same patient. 
The use of ${ }^{11} \mathrm{C}$-carbon monoxide in new applications within a mini clean room and on new technology platforms has been demonstrated. The mini-clean room simplifies GMP production and will facilitate the access to various tracers.

VAAT null mutant mice has proven to be an animal model with altered sensorimotor gating, a symptom associated to schizophrenia. Higher binding potential of ${ }^{11} \mathrm{C}$-raclopride has been measured with this model compared to wild type control animals.

We may conclude that the tools developed within the MINDVIEW project will facilitate and extend the technique of dynamic and simultaneous PET and MR imaging, opening new vistas for understanding mental disorders. It will enable the full breath of activation paradigms to be used. These include dynamic imaging using activation paradigms as the perturbation method for studying the brain from several aspects: morphological, functional, metabolic, neurotransmitter and neuro-receptor effects. The ability to perform serial studies, i.e., examinations within the same subject, paves the way for personalized medicine such as early diagnosis of disease onset and therapy monitoring.

\section{Acknowledgements}

This project is funded by EU grant FP7-HEALTH-F2-2013-603002.

\section{References}

[1] Michelgård A, Appel L, Pissiota A, et al., Symptom provocation in specific phobia affects the substance P neurokinin-1 receptor system, Biol Psychiatry. 61(8), pp. 1002-1006, 2007.

[2] Howes OD, Kambeitz J, Kim E, et al., The Nature of Dopamine Dysfunction in Schizophrenia and What This Means for Treatment, Arch Gen Psychiatry, 2012.

[3] Farde L, Nordström A, Wiesel F, et al., Positron Emission Tomographic Analysis of Central D1 and D2 Dopamine Receptor Occupancy in Patients Treated with Classical Neuroleptics and Clozapine, Arch. Gen. Psychiatry 49, 538, 1992.

[4] Krystal JH, Karper LP, Seibyl JP, et al., Subanesthetic effects of the noncompetitive NMDA antagonist, ketamine, in humans. Psychotomimetic, perceptual, cognitive, and neuroendocrine responses, Arch Gen Psychiatry 51, 199, 1994.

[5] Howes O, McCutcheon R and Stone J, Glutamate and dopamine in schizophrenia: An update for the 21st century, Journal of Psychopharmacology, 2015. 
[6] Attucci S, Carlà V, Mannaioni G and Moroni F, Activation of type 5 metabotropic glutamate receptors enhances NMDA responses in mice cortical wedges, Br J Pharmacol 132, 799, 2001.

[7] Ametamey SM, Treyer V, Streffer J, et al., Human PET Studies of Metabotropic Glutamate Receptor Subtype 5 with ${ }^{11}$ C-ABP688, J Nucl Med 48, 247, 2007.

[8] Sullivan JM, Lim K, Labaree D, et al., Kinetic analysis of the metabotropic glutamate subtype 5 tracer $\left[{ }^{18} \mathrm{~F}\right] \mathrm{FPEB}$ in bolus and bolusplus-constant-infusion studies in humans, J Cereb Blood Flow Metab 33, 532, 2013.

[9] Wong DF, Waterhouse R, Kuwabara H, et al., ${ }^{18}$ F-FPEB, a PET Radiopharmaceutical for Quantifying Metabotropic Glutamate 5 Receptors: A First-in-Human Study of Radiochemical Safety, Biokinetics, and Radiation Dosimetry, J Nucl Med 54, $388,2013$.

[10] Young JW, Head and Face Antropometry of Adult U.S. Citizens, Civil Aeromedical Institute, Federal Aviation Administration, U.S. Department of Transportation, Report number DOT/FAA/AM-93/10, July 1993.

[11]Collins CM, Li S, Yang QX and Smith MB, A Method for Accurate Calculation of B1 Fields in Three Dimensions. Effects of Shield Geometry on Field Strength and Homogeneity in the Birdcage Coil, Journal of Magnetic Resonance 125, pp. 233-241, 1997.

[12]Ribeiro PJ, Cricenti SV and Lancellotti CLP, Encephalometry on the medial face of the human brain hemisphere, A necropsy study, Arq Neuropsiquiatr 63(2-B), pp. 430-436, 2005.

[13] Gonzalez AJ, Majewski S, Sánchez F, et al., The MINDVIEW brain PET detector, feasibility study based on SiPM arrays, Nucl. Instrum. Meth. A 818, pp. 81, 2016.

[14] Sánchez F, Benlloch JM, Escat B, et al., Design and test of a portable mini gamma camera, Med. Phys. 31, 1384-1397, 2004.

[15]Levin CS, Detector design issues for compact nuclear emission cameras dedicated to breast imaging, Nucl. Instrum. Methods A 497 (2003) 60-74.

[16]Ling T, Depth of interaction decoding of a continuous crystal detector module, Phys. Med. Biol. 52, 2213-2228, 2007.

[17]Vaska P, Krishnamoorthy S, Stoll S, et al., An improved Anger detector approach for PET with high resolution and sensitivity, IEEE Nucl. Sci. Symp. Med. Img. Conf. 6, 3463-3466, 2004.

[18]Antich P, Malakhov N, Parkey R, Slavin N, and Tsyganov E, 3D position readout from thick scintillators, Nucl. Instrum. Methods A 480, $782-787$ (2002).

[19]Gonzalez-Montoro A, Aguilar A, Cañizares G, et al., Performance Study of a Large Monolithic LYSO PET Detector With Accurate Photon DOI Using Retroreflector Layers, IEEE Trans. Rad. Plasma Med. Sci. vol 1, pp. 229, 2017.

[20]Pani R, Bettiol M, Preziosi E, et al., A Novel Method for gamma-photons Depth-of-Interaction Detection in Monolithic Scintillation Crystals, IEEE Transactions on Nuclear Science, 63(5), 2487-2495, 2016.

[21] Kaul M, Surti S, and Karp JS, Combining surface treatments with shallow slots to improve the spatial resolution performance of continuous, thick LYSO detectors for PET, IEEE Trans. Nucl. Sci., vol. 60, no. 1, pp. 44-52, Feb. 2013. 
[22]Jackson C, O’Neill K, Wall N, and McGarvey B, High-volume silicon photomultiplier production, performance, and reliability, Opt. Eng., vol. 53, no. 8, 2014, Art. no. 081909.

[23] González AJ, Sanchez F, Conde P, et al., A novel brain PET insert for the MINDView project, 2014 IEEE Nuclear Science Symposium and Medical Imaging Conference (NSS/MIC), Seattle, WA, 2014, pp. 1-4.

[24]Preziosi E, Sánchez S, González AJ, et al., Performance study of a PET scanner based on monolithic scintillators for different DoIdependent methods, Journal of Instrumentation 11(12), C12076, 2016.

[25]Pani R, Bettiol M, Preziosi E, et al., Position algorithm for monolithic scintillation crystals based on charge projection readout, Journal of Instrumentation, 11(01), C01061, 2016.

[26]Långström B and Sjöberg CO, System for controlling environment in reaction box, From PCT Int. Appl. (2013), WO 2013103312 A1 20130711.

[27]Rahman O, Langstroem B, and Halldin C, Alkyl Iodides and $\left[{ }^{11} \mathrm{C}\right] \mathrm{CO}$ in Nickel-Mediated Cross-Coupling Reactions: Successful Use of Alkyl Electrophiles containing a $\beta$ Hydrogen Atom in Metal-Mediated $\left[{ }^{11} \mathrm{C}\right]$ Carbonylation, ChemistrySelect $1(10), 2498-2501,2016$.

[28]Dahl K, Schou M, Ulin J, Sjoeberg C-O, Farde L, Halldin C, ${ }^{11}$ C-Carbonylation reactions using gas-liquid segmented microfluidics, RSC Advances 5(108), 88886-88889, 2015.

[29]Eriksson J, Ulin J, and Långström B, $\left[{ }^{11} \mathrm{C}\right]$ Methyl iodide from $\left[{ }^{11} \mathrm{C}\right] \mathrm{methane}$ and iodine using a non-thermal plasma method, J. Label. Comp. and Radiopharm. 49(13), 1177-1186, 2006.

[30]Rahman O, Takano A, Amini N, et al., Synthesis of $\left({ }^{11} \mathrm{C}\right)$ carbonyl Raclopride and $\left({ }^{11} \mathrm{C}\right.$-methyl)Raclopride in a Monkey PET study, Nuclear Medicine and Biology 42(11), 893-898, 2015.

[31]González AJ, Gonzalez-Montoro A, Aguilar A, et al., A brain PET insert MR compatible: Final design and first results, 2016 IEEE Nuclear Science Symposium, Medical Imaging Conference and Room-Temperature Semiconductor Detector Workshop (NSS/MIC/RTSD), Strasbourg, 2016, pp. 1-5.

[32]Autret A, Bert J, Strauss O and Visvikis D, Projector with realistic detector scatter modelling for PET list-mode reconstruction, 2012 IEEE Nuclear Science Symposium and Medical Imaging Conference Record (NSS/MIC), Anaheim, CA, 2012, pp. $3333-3336$.

[33]Zelano J, Mikulovic S, Patra K, et al., The synaptic protein encoded by the gene Slc10A4 suppresses epileptiform activity and regulates sensitivity to cholinergic chemoconvulsants, Exp. Neurol. 239, 73-81, 2013.

[34]Larhammar M, Patra K, Blunder M, et al., SLC10A4 is a vesicular amine-associated transporter modulating dopamine homeostasis, Biol. Psychiatry 77(6), 526-36, 2015.

[35]Patra K, Lyons DJ, Bauer P, et al., A role for solute carrier family 10 member 4, or vesicular aminergic-associated transporter, in structural remodelling and transmitter release at the mouse neuromuscular junction, Eur. J. Neurosci. 41(3), 316-27, 2015.

[36]Braff DL and Geyer MA, Sensorimotor gating and schizophrenia. Human and animal model studies, Arch. Gen. Psychiatry 47(2), 181-8, 1990. 
[37]Farde L, Wiesel F-A, Stone-Elander S, et al., $\mathrm{D}_{2}$ dopamine receptors in neuroleptic-naive schizophrenic patients, Arch. Gen. Psychiatry $47,213-219,1990$

[38]Blelch A, Brown S-L, Kahn R and van Praag H-M, The Role of Serotonin in Schizophrenia, Schizophrenia Bulletin 14(2), $297,1988$.

[39]Finnema SJ, Stepanov V, Ettrup A, et al., Characterization of $\left[{ }^{11} \mathrm{C}\right] \mathrm{Cimbi}-36$ as an agonist PET radioligand for the 5-HT2A and 5-HT2C receptors in the nonhuman primate brain, NeuroImage 84, 342-353, 2014.

[40]Collste K, Plavén-Sigray P, Fatouros-Bergman H, et al., Lower levels of the glial cell marker TSPO in drug-naive first-episode psychosis patients as measured using PET and $\left[{ }^{11} \mathrm{C}\right] \mathrm{PBR} 28$, Molecular Psychiatry 22, 850-856, 2017. 